\title{
Adaptive symmetric mean filter: a new noise-reduction approach based on the slope facet model
}

\author{
Huan-Chao Huang, Chung-Ming Chen, Sheng-De Wang, and Henry Horng-Shing Lu
}

\begin{abstract}
Two new noise-reduction algorithms, namely, the adaptive symmetric mean filter (ASMF) and the hybrid filter, are presented in this paper. The idea of the ASMF is to find the largest symmetric region on a slope facet by incorporation of the gradient similarity criterion and the symmetry constraint into region growing. The gradient similarity criterion allows more pixels to be included for a statistically better estimation, whereas the symmetry constraint promises an unbiased estimate if the noise is completely removed. The hybrid filter combines the advantages of the ASMF, the double-window modified-trimmed mean filter, and the adaptive mean filter to optimize noise reduction on the step and the ramp edges. The experimental results have shown the ASMF and the hybrid filter are superior to three conventional filters for the synthetic and the natural images in terms of the root-mean-squared error, the root-meansquared difference of gradient, and the visual presentation. (C) 2001 Optical Society of America

OCIS codes: $100.0100,030.4280,100.2980$.
\end{abstract}

\section{Introduction}

Noise reduction is a process to remove the noise from a corrupted image and preferably keep the structures of the original image simultaneously. Because the noise not only degrades the visual resolution but also impedes the quantitative analysis of an image, noise reduction has been considered an essential task for many image-processing applications. To eliminate the noise while preserving the image structures, an enormous number of noise-reduction algorithms, ranging from simple averaging to regularization approaches, have been proposed in the past.

A noise-reduction algorithm usually comprises three essences, namely, image model, denoising window, and denoising strategy. The image model characterizes the intensity variation of adjacent pixels, which serves as the basis of the design of a noisereduction algorithm. Some widely used models are the flat facet model, ${ }^{1}$ the slope facet model, ${ }^{1}$ and the

H.-C. Huang and S.-D. Wang are with the Institute of Electrical Engineering, National Taiwan University, Taipei, Taiwan. C.-M. Chen (e-mail address: ming@lotus.mc.ntu.edu.tw), the corresponding author, is with the Institute of Biomedical Engineering, National Taiwan University, Taipei, Taiwan. H. H.-S. Lu is with the Institute of Statistics, National Chiao-Tung University, HsinChu, Taiwan.

Received 1 February 2001; revised manuscript received 13 July 2001.

0003-6935/01/295192-14\$15.00/0

(C) 2001 Optical Society of America piecewise polynomial model. ${ }^{2}$ For each pixel to be denoised, called a target pixel, the denoising window defines the region from which the neighborhood information is utilized by the denoising strategy. For convenience, the pixels in the denoising window, excluding the target pixel, are called window pixels. The shape of the denoising window may be regular or irregular. The shape and the size of the denoising window may be fixed for all target pixels or may be changed adaptively subject to specific criteria. Note that the denoising window is not necessarily a continuous region. It may be constituted by a set of pixels sporadically distributed in the vicinity of the target pixel. As the core of a noise-reduction algorithm, the denoising strategy attempts to attain an estimate, which is as close to the original (uncorrupted) image as possible, using the information provided in every denoising window. Four typical types of denoising strategies are the order statistic filters, ${ }^{3}$ the weighted mean filters, ${ }^{4}$ the regression filters, ${ }^{2}$ and the regularization filters..$^{5,6}$

Most early noise-reduction algorithms were implicitly or explicitly designed on the basis of the flat facet model. The flat facet model assumes that the original image is composed of horizontal planes, and adjacent planes are connected by the step edges. To preserve the step edges, two types of denoising strategies have been employed by these noise-reduction algorithms. In a denoising window the first type of denoising strategies tends to give a larger weight to a pixel that is less likely to be an edge point during 
integration of the neighborhood information, e.g., computation of the weighted sum of the gray levels of all pixels in the window. One example is the gradient inverse weighted smoothing scheme proposed by Wang and Vagucci, ${ }^{7}$ in which the gray level of each target pixel is replaced by the weighted sum of the gray levels of all pixels in the corresponding denoising window. The denoising window is a $3 \times 3$ pixel window centered at each target pixel. The weight of each window pixel is inversely proportional to the magnitude of the gradient at that pixel. Another example is the filter proposed by Lee, ${ }^{8}$ in which the gray level of the target pixel is substituted by the weighted sum of the gray level of the target pixel and the mean of the gray levels of the window pixels. A larger weight is assigned to a target pixel with a larger variance, which may be estimated from the neighboring pixels of the target pixel. The denoising window is a square window of a fixed size.

In the second type of denoising strategies the denoising window contains only those window pixels that are approximately on the same horizontal plane as the corresponding target pixel or the selected reference pixel. For instance, the adaptive mean filter ${ }^{4}$ (AMF) selects the window pixels in such a way that the differences between the window pixels and the target pixel must be less than a specified threshold. The double-window modified-trimmed mean (DWMTM) filter ${ }^{9,10}$ chooses the window pixels with the constraint that the differences between the window pixels in the large window and the median of the small window, which is centered at the target pixel, are limited to a prescribed range. The adaptive neighborhood noise subtraction (ANNS) filter ${ }^{11}$ not only requires the window pixels to be roughly on the same horizontal plane as the target pixel but also adopts Lee's approach ${ }^{8}$ to estimate the gray level of the target pixel.

Although the noise-reduction algorithms based on the flat facet model may have fast denoising strategies, they share an inherent weakness, which is that the assumed step edges are rarely found in natural images. Because of the system point-spread function embedded in an imaging system, in reality, most natural image edges generally appear as linear or nonlinear ramp edges with various inclinations. As a consequence, these noise-reduction algorithms tend to distort the structures that are not linear or the areas at which the local extrema occur. Furthermore, both types of denoising strategies are expected to be ineffective for noises on the ramp edges because of the large variances in the denoising windows and the small areas of the attainable horizontal planes, both resulting from the ramp structures.

To ameliorate the deficiency of the flat facet model, many alternative image models have been employed previously to describe natural image edges. Following Acton and Bovik, ${ }^{5}$ most of them may be categorized into two classes, i.e., the piecewise image models (PIMs) and the local image models (LIMs). The PIM models images as obeying an image property such as constancy, linearity, or some other more complex property over the entire image in a piecewise manner. Note that the flat facet model is a PIM of zero order. For differentiation, PIMs other than the flat facet model are called the high-order PIMs. The LIM models images as obeying an image property such as monotonicity, convexity-concavity, or some other desired property for every denoising window within the image. To satisfy the constraints of the specified image properties, the noise-reduction algorithms based on these image models generally adopt such computation-intensive denoising strategies as linear-nonlinear regression, regularization, and so on. As a result, these noise-reduction algorithms are usually time-consuming and may not be practical for real-time applications, even though the highorder PIMs and the LIMs may approximate the natural image edges more closely than the flat facet model.

Another problem that the high-order PIMs and the LIMs may have is the difficulty in determining the proper shape and size of the denoising window. For those noise-reduction algorithms based on the PIMs and the LIMs, to fit the desired image models, the denoising strategies, e.g., the regularization techniques, usually incorporate denoising windows with regular shapes, e.g., a square window. Statistically, a denoising strategy prefers a large denoising window to a small one because using more neighborhood information is more likely to attain a better estimation, provided that the pixels in the window fulfill the desired image model. However, denoising with a large regular-shape window is apt to distort the image structures because a regular-shape window may inevitably contain pixels belonging to different pieces, especially when the window is large. Even though a better window size may be found by testing multiple window sizes and choosing the best one, ${ }^{12,13}$ the induced higher computational cost is usually undesirable. Besides, the fixed window shape would intrinsically hinder the noise-reduction algorithms from maximizing the number of pixels included in each denoising window.

Ideally, the denoising window for each target pixel should include as many neighboring pixels that share the specified image property with the target pixel as possible. The shape and the size of the ideal denoising window would naturally vary with the target pixel. Several techniques ${ }^{9,11,14,15}$ have been proposed to adaptively determine the set of pixels for each denoising window, whereas they were designed for the flat facet model and cannot be used for the high-order PIMs and the LIMs, which are usually preferred to approximate the natural image edges more closely.

To optimize utilization of the neighborhood information while using a image model more realistic than the flat facet model, in this paper we propose two new noise-reduction algorithms for removing noises with a constant mean over the entire image. The first one is a new adaptive noise-reduction algorithm, called the adaptive symmetric mean filter (ASMF), which features the slope facet model, the adaptive denoising 
window, and the fast noniterative denoising strategy. The slope facet model, though not the optimal model, is a reasonable choice, considering the implementation complexity and the closeness of the model to the natural image edges. On the basis of the slope facet model, we have developed a new symmetric regiongrowing scheme to adaptively find the denoising window in which the simple mean filter is employed to remove the noises.

Whereas the first algorithm is designed to model the ramp edges, the second one is to account for the wide spectrum of the slope of the natural ramp edges. The second algorithm is a hybrid algorithm combining the advantages of the proposed ASMF and two conventional algorithms, namely, the DWMTM9,10 filter and the AMF. 4 The idea is to use the ASMF for a slowly varying ramp edge and the conventional algorithms for a steep ramp edge. The rationale behind the hybrid algorithm is that when the ramp edge is steep, the limited number of pixels contained in the denoising window may result in a poor statistic property, and in this case it would be more appropriate to perform denoising based on the flat facet model. However, according to the study of Fong et al., ${ }^{14}$ the DWMTM filter and the AMF have a great edgepreserving capability in removing noises around the step edges. Moreover, the AMF is better in preserving the lines and the corners, whereas the DWMTM filter is superior when the signal-to-noise ratio is low around the target pixel.

The proposed algorithms will be compared with three conventional noise reduction algorithms, namely, the DWMTM filter, the AMF, and the ANNS filter. Two types of images are used, i.e., the synthetic and the natural images. The synthetic images are blurred with Gaussian filters of different standard deviations to simulate the ramp edges. The noises are additive white Gaussian noises of various standard deviations with a zero mean.

This paper is organized as follows. Section 2 describes the two proposed algorithms, the ASMF and the hybrid filter, as well three conventional algorithms, the DWMTM filter, the AMF, and the ANNS filter. Section 3 presents the experimental results and discussions. The conclusions are given in Section 4.

\section{The Noise-Reduction Algorithms}

Five noise-reduction algorithms, the DWMTM filter, the AMF, the ANNS filter, the ASMF, and the hybrid filter, are described in this section. The first three are the conventional algorithms to be compared with the proposed ASMF and hybrid algorithms. The DWMTM filter, the AMF, and the ANNS filter are selected to represent three commonly used approaches to determining the adaptive denoising window for each target pixel. The DWMTM filter and the $\mathrm{AMF}$, which have been shown to have strong ability in removing Gaussian noises, ${ }^{14}$ are typical examples of using thresholding techniques to choose the window pixels within a predefined size of window. The difference between these two filters is the DW-
MTM filter uses a smaller window to estimate the reference for thresholding and the AMF uses the target pixel as the reference. The ANNS filter stands for the class of algorithms employing the regiongrowing scheme to find a connected set of pixels with similar gray levels. Besides, all these three filters share a common characteristic, which is that the denoising strategies are noniterative. The reasons why the iterative denoising strategies are not considered in the comparative study are twofold. One reason is that it is not fair to compare the performance between iterative and noniterative denoising strategies. The other reason is that the iterative denoising strategy usually requires a long processing time, which is not desirable for many real-time applications.

A set of pixels are defined as intensity irrelevant if this set is formed without imposing any constraint on the pixel intensities. Otherwise, these pixels are referred to as intensity relevant. For succinctness of presentation, the notations defined below will be used throughout this paper.

$W_{k}$ : the set of pixels in the denoising window of a target pixel $p_{k}$;

$S\left(p_{i}\right)$ : the signal portion of the gray level of the pixel $p_{i}$;

$N\left(p_{i}\right)$ : the noise portion of the gray level of the pixel $p_{i}$;

$I\left(p_{i}\right)$ : the gray level of the pixel $p_{i}$, which is equal to $S\left(p_{i}\right)+N\left(p_{i}\right)$;

$S\left(W_{k}\right)$ : the set of $S\left(p_{i}\right)$ 's of all pixels in $W_{k}$, i.e., $\left\{S\left(p_{i}\right) \mid \forall p_{i} \in W_{k}\right\}$;

$N\left(W_{k}\right)$ : the set of $N\left(p_{i}\right)$ 's of all pixels in $W_{k}$, i.e., $\left\{N\left(p_{i}\right) \mid \forall p_{i} \in W_{k}\right\}$;

$I\left(W_{k}\right)$ : the set of gray levels of all pixels in $W_{k}$, i.e., $\left\{I\left(p_{i}\right) \mid \forall p_{i} \in W_{k}\right\}$

$\varepsilon_{k}$ : the estimation error for a target pixel $p_{k}$, i.e., the difference between $S\left(p_{k}\right)$ and the output of a filter for $p_{k}$;

$E(X):$ the mean of $X$

$V(X)$ : the variance of $X$.

It is assumed in this paper that the noise is additive, uncorrelated with the image, and that the means of the noises at all pixels in the image are the same, i.e., $E\left[N\left(p_{k}\right)\right]=\mu, \forall p_{k}$. Furthermore, the noise is assumed to be ergodic, i.e., $E\left[N\left(W_{k}\right)\right]=$ $E\left[N\left(p_{k}\right)\right]=\mu$, provided that the pixels in $W_{k}$ are intensity irrelevant. Without loss of generality, $\mu$ is set to 0 in the following discussions. Note that the ergodic property is valid only for the set of pixels that are intensity irrelevant because the constraints imposed on the pixel intensities might bias the mean of the gray levels of the pixels in the set.

\section{A. Double-Window Modified-Trimmed Mean Filter}

The DWMTM filter has great ability in noise reduction and edge preserving but poor ability in line preservation. ${ }^{14}$ For each target pixel, $p_{k}$, the DWMTM filter defines the noise-reduction operation by use of two windows, $D S W_{k}$ and $D L W_{k}$, centered at $p_{k}$, where 
the size of $D S W_{k}$ is smaller than that of $D L W_{k}$. Let $m_{k}$ stand for the median of the pixel gray levels in $D S W_{k}$. Then the denoising window of $p_{k}$ is defined by

$$
W_{k}=\left\{p_{i}|| I\left(p_{i}\right)-m_{k} \mid<T_{\mathrm{dwmtm}}, \quad \forall p_{i} \in D L W_{k}\right\},
$$

where $T_{\text {dwmtm }}$ is a predetermined threshold. This criterion has implicitly assumed the flat facet model for the DWMTM filter. It is clear that the denoising window of the DWMTM filter is not necessary a single-connected component. Finally, the output of the DWMTM filter for $p_{k}$ is defined as the mean of the pixel gray levels in $W_{k}$, i.e., $E\left[I\left(W_{k}\right)\right]$.

\section{B. Adaptive Mean Filter}

Compared with the DWMTM filter, the AMF is much better in line preserving and slightly worse in noise reduction. ${ }^{14}$ Both have good performance in edge preserving. ${ }^{14}$ In contrast to the DWMTM filter that uses double windows, the AMF employs only one window, $A W_{k}$, to define the noise-reduction operation. For each target pixel, $p_{k}$, the denoising window is defined by

$$
W_{k}=\left\{p_{i} \| I\left(p_{i}\right)-I\left(p_{k}\right) \mid<T_{\text {amf }}, \forall p_{i} \in A W_{k}\right\} .
$$

Then the output of the AMF for $p_{k}$ is defined as the mean of the pixel gray levels in $W_{k}$, i.e., $E\left[I\left(W_{k}\right)\right]$. Like the DWMTM filter, the flat facet model has been implicitly assumed and the denoising window of the AMF is not necessary a single-connected component.

\section{Adaptive-Neighborhood Noise Subtraction Filter}

Unlike the DWMTM filter and the AMF, the ANNS filter ${ }^{11}$ takes advantage of the region-growing technique to derive a connected denoising window for each target pixel, $p_{k}$. Using each target pixel, $p_{k}$, as the seed, the denoising window for $p_{k}$ will include every pixel $p_{i}$ that is eight-connected to the seed and satisfies $\left|I\left(p_{k}\right)-I\left(p_{i}\right)\right|<T_{\text {anns. }}$. The total number of pixels in a denoising window is limited to a predefined number, $Q_{\text {anns }}{ }^{2}$. Then the estimate of $S\left(p_{k}\right)$ is

$$
\begin{aligned}
y_{k}= & E\left[I\left(W_{k}\right)\right]+\left[1-\left(\frac{\sigma_{n}{ }^{2}}{{\sigma_{d^{\prime}}}^{2}+{\sigma_{n}}^{2}}\right)^{1 / 2}\right] \\
& \times\left\{I\left(p_{k}\right)-E\left[I\left(W_{k}\right)\right]\right\},
\end{aligned}
$$

where $\sigma_{n}{ }^{2}$ is the variance of the noises, which is assumed to be known and $\sigma_{d^{\prime}}{ }^{2}$ is the estimate of $V\left[S\left(W_{k}\right)\right]$, which is defined as $V\left[I\left(W_{k}\right)\right]-\sigma_{n}^{2}$.

Two kinds of denoising windows were considered by Paranjape et al. ${ }^{11}$ The ANNS filter first uses a $Q_{\text {anns }} \times Q_{\text {anns }}$ square window, which is centered at each target pixel, $p_{k}$, to estimate ${\sigma_{d^{\prime}}}^{2}$. If ${\sigma_{d^{\prime}}}^{2}<{\sigma_{n}}^{2}$, the square window is used as the denoising window in Eq. (3) to estimate $S\left(p_{k}\right)$. Otherwise, a new denoising window is found by region growing with a threshold $T_{\text {anns }}=2 \sigma_{d^{\prime}}$ for Eq. (3). The new $\sigma_{d^{\prime}}{ }^{2}$

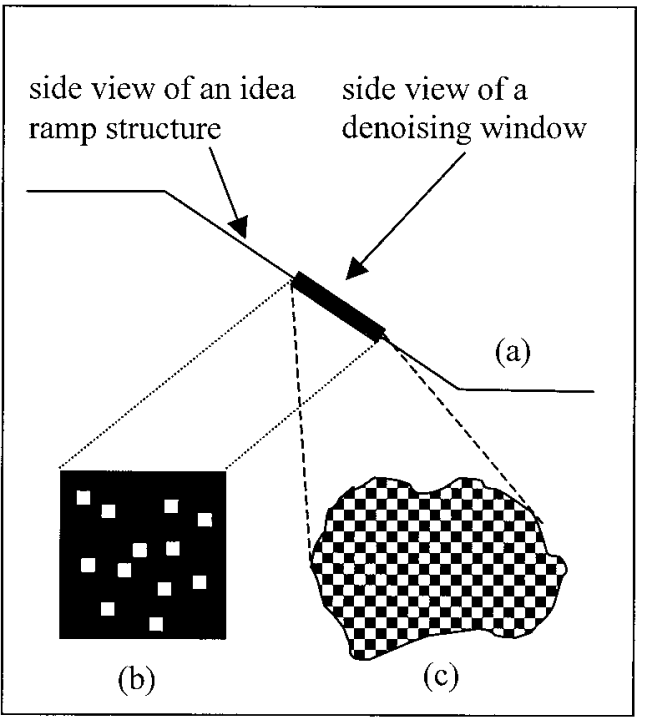

Fig. 1. (a) Side view of an ideal ramp edge. (b) The front view of the denoising window, derived with the DWMTM filter or the AMF, for a target pixel on the ideal ramp edge. (c) The front view of the denoising window, derived with the ANNS filter, for a target pixel on the ideal ramp edge.

associated with the new denoising window is calculated as

$$
\sigma_{d^{\prime}}{ }^{2}=\left\{\begin{array}{ll}
V\left[I\left(W_{k}\right)\right]-\sigma_{n}{ }^{2} & \text { for } V\left[I\left(W_{k}\right)\right]>\sigma_{n}{ }^{2} \\
0 & \text { for } V\left[I\left(W_{k}\right)\right] \leq \sigma_{n}{ }^{2}
\end{array} .\right.
$$

\section{Proposed Adaptive Symmetric Mean Filter}

By gathering the pixels with the similar gray levels to the reference pixel, the DWMTM filter, the AMF, and the ANNS filter have inherently assumed the flat facet model for the image. Despite of their outstanding performances on the flat areas, these three filters suffer at least two potential problems on the ramp structures, which are monotonically increasing or decreasing. Consider an idea ramp structure, which is defined as a set of connected pixels with the same normal vector everywhere on the surface formed by the intensities of these pixels. As an example, the side view of an ideal ramp structure is shown in Fig. 1(a). For a target pixel on the ramp structure, its typical denoising window derived by the DWMTM filter or the AMF is illustrated in Fig. 1(b), and that derived by the ANNS filter is given in Fig. 1(c).

Because the threshold used in these three filters should be kept reasonably small to avoid smearing out the nonflat structures, one potential problem of these three filters is the limited number of window pixels that could be included in a denoising window on a ramp structure. It suggests that the outputs of the filters may be statistically unreliable. The other problem arises from the fact that $E\left[S\left(W_{k}\right)\right]$ does not guarantee to be equal to $S\left(p_{k}\right)$ because of the potentially asymmetric spatial distribution of the window pixels found by these three filters as illustrated in Figs. 1(b) and 1(c). It means that 


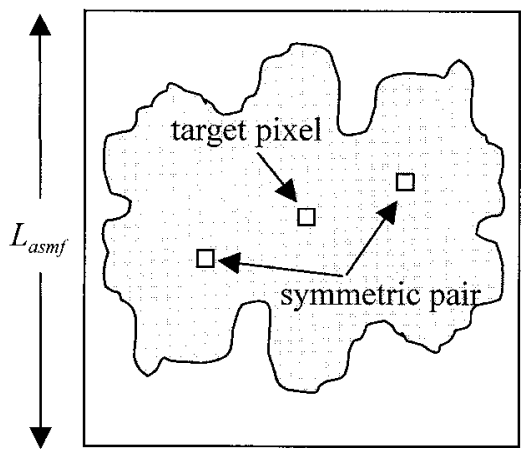

Maximum region size $L_{\text {asmf }} \times L_{\text {asm }}$

Fig. 2. Symmetric denoising window derived with the ASMF, in which the central square is the target pixel of the denoising window and the other two squares represent a pair of pixels symmetric with respect to the target pixel.

these three filters do not promise a correct estimate for an ideal ramp structure even if the denoising window is noise free and contains a sufficient number of window pixels.

To amend these two problems inherent in the DWMTM filter, the AMF, and the ANNS filter, a new noise-reduction algorithm based on the slope facet model, called the ASMF, is proposed in this paper. The goal of the proposed ASMF is to find as many pixels as possible for a denoising window not only on a flat area but also on a ramp area and to ensure the correctness of the estimate within a reasonable computation time. To achieve this goal, the proposed ASMF models the image as a composition of nonoverlapped slope facets, each of which may be considered an ideal ramp. For each target pixel $p_{k}$, the ASMF attempts to find the largest symmetric denoising window $W_{k}$ by use of the region-growing technique such that all pixels in the denoising window are on the same slope facet. The size of $W_{k}$ is bounded by the maximum region size $L_{\text {asmf }} \times L_{\text {asmf }}$. By symmetry, we mean that for every window pixel $p_{i}$ in the denoising window, there exists another window pixel $p_{j}$ such that $p_{i}$ and $p_{j}$ are symmetric with respect to $p_{k}$. As an example, a symmetric denoising window is illustrated in Fig. 2, in which the central square is the target pixel of the denoising window and the other two squares represent a pair of pixels symmetric with respect to the target pixel.

To ensure that all pixels in the denoising window are on the same slope facet, we propose that the gradient similarity serve as the criterion for region growing, instead of the common criterion, the intensity similarity, employed by many other regiongrowing-based approaches such as the ANNS filter. The gradient threshold used in the region growing is denoted as $T_{\text {asmf }}$. To reduce the estimation error of the gradient possibly introduced by the noise, the regional gradient derived with the horizontal and vertical Kirsch templates ${ }^{16}$ is used in this study instead of the widely-used local gradient computed from the eight nearest neighbors of each pixel. The horizontal Kirsch template of size $W_{\text {kirsch }} \times W_{\text {kirsch }}$ is defined as

$\left\{[-1]_{W_{\text {kirsch }} \times\left[\left(W_{\text {kirsch }}-1\right) / 2\right]}[0]_{W_{\text {kirsch }} \times 1}[1]_{W_{\text {kirsch }} \times\left[\left(W_{\text {kirsch }}-1\right) / 2\right]}\right\}$, where $[a]_{m \times n}$ represents an $m \times n$ matrix with all elements equal to $a$. The vertical Kirsch template is the transpose of the horizontal Kirsch template of the same size. Because the gradient estimation, though important, is not the focus of this paper, the Kirsch templates have been chosen only for their simplicity and variety of window sizes. One may use any other gradient estimator to derive a better estimation of the gradient for each pixel.

Once the symmetric denoising window $W_{k}$ is found for a target pixel $p_{k}$, the output of the ASMF is defined as the mean of the pixel gray levels in $W_{k}$, i.e., $E\left[I\left(W_{k}\right)\right]$. It is expected that the proposed ASMF will make a correct estimation for any target pixel $p_{k}$ on the ideal ramp structure and on the flat area because for both types of structures,

$$
\begin{aligned}
E\left[I\left(W_{k}\right)\right] & =E\left[S\left(W_{k}\right)\right]+E\left[N\left(W_{k}\right)\right] \\
& =E\left[S\left(W_{k}\right)\right] \\
& =S\left(p_{k}\right) .
\end{aligned}
$$

From line one to line two of Eq. (4), $E\left[N\left(W_{k}\right)\right]$ is set to 0 on the basis of the ergodic property. In lines two and three $E\left[S\left(W_{k}\right)\right]=S\left(p_{k}\right)$ is ensured by the symmetry property of the denoising window $W_{k}$.

In comparison with the DWMTM filter, the AMF, and the ANNS filter, the proposed ASMF is expected to have a better performance than these three filters on the ramp structure with a gentle slope; the ASMF not only finds more pixels in the denoising window by use of the gradient similarity but also makes certain that every window pixel can be paired up with another window pixel, both of which are symmetric with respect to the target pixel. The former gives a statistically more plausible result, whereas the latter ensures $E\left[S\left(W_{k}\right)\right]=S\left(p_{k}\right)$. Nevertheless, when the ramp structure has a steep slope, the estimation derived by the ASMF becomes less reliable owing to the limited number of pixels that can be found on a steep ramp. One exception is if the steep ramp edge is relatively long, then for any target pixel on the ramp, the ASMF might be able to find a long strip of denoising window in parallel with the steep ramp edge.

The proposed ASMF is also expected to outperform the DWMTM filter, the AMF, and the ANNS filter on the flat areas. According to Eq. (4), the estimation error caused by the proposed ASMF would be

$$
\begin{aligned}
\varepsilon_{k} & =E\left[I\left(W_{k}\right)\right]-S\left(p_{k}\right) \\
& =0 .
\end{aligned}
$$

Nevertheless, for both the DWMTM filter and the $\mathrm{AMF}$, the estimation error for a target pixel $p_{k}$ on a flat area is given by

$$
\begin{aligned}
\varepsilon_{k} & =E\left[I\left(W_{k}\right)\right]-S\left(p_{k}\right) \\
& =E\left[N\left(W_{k}\right)\right],
\end{aligned}
$$




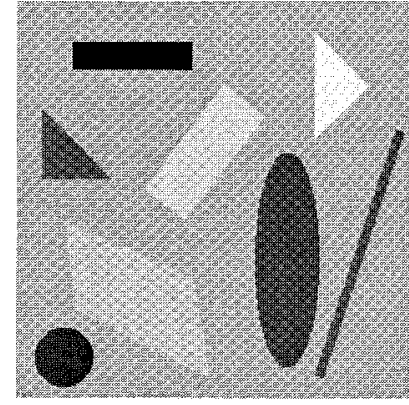

(a)

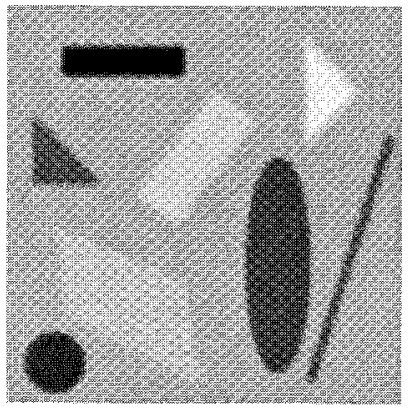

(b)
Fig. 3. Uncorrupted synthetic images with (a) $\sigma_{b}=0$ and (b) $\sigma_{b}=2$.

with $E\left[S\left(W_{k}\right)\right]=S\left(p_{k}\right)$ for a flat area. Because the pixels in $W_{k}$ are intensity relevant, the ergodic property no longer holds for $W_{k}$, which means that $E\left[N\left(W_{k}\right)\right]$ would not be 0 in general. Although it is hard to determine the estimation errors without knowing the probability density function of the noise, it can be shown that the estimation errors are bounded subject to the intensity constraints. More specifically, for the DWMTM filter

$$
-T_{\mathrm{dwmtm}}+\delta_{k}<\varepsilon_{k}<T_{\mathrm{dwmtm}}+\delta_{k}
$$

where $\delta_{k}=m_{k}-S\left(p_{k}\right)$, and for the AMF

$$
-T_{\mathrm{amf}}+N\left(p_{k}\right)<\varepsilon_{k}<T_{\mathrm{amf}}+N\left(p_{k}\right) .
$$

For the ANNS filter, because $\sigma_{d^{\prime}}{ }^{2}$ is expected to be small for a flat facet, the $Q_{\text {anns }} \times Q_{\text {anns }}$ square window would most probably be used as the denoising window. In this case, because all pixels in the window are intensity irrelevant, the ergodic property may be applied, i.e., $E\left[N\left(W_{k}\right)\right]=0$, which leads to

$$
\varepsilon_{k}=\left[1-\left(\frac{\sigma_{n}^{2}}{{\sigma_{d^{\prime}}}^{2}+\sigma_{n}^{2}}\right)^{1 / 2}\right] N\left(p_{k}\right) .
$$

If ${\sigma_{d^{\prime}}}^{2} \ll{\sigma_{n}}^{2}$, by use of the Taylor expansion, $\varepsilon_{k}$ may be further reduced to

$$
\varepsilon_{k}=\left\{1-\left[\frac{1}{1+\left(\sigma_{d^{\prime}}{ }^{2} / \sigma_{n}{ }^{2}\right)}\right]^{1 / 2}\right\} N\left(p_{k}\right) \approx \frac{\sigma_{d^{\prime}}{ }^{2}}{2 \sigma_{n}{ }^{2}} N\left(p_{k}\right) .
$$

\section{E. Hybrid Filter}

By use of the gradient similarity criterion and imposing the symmetry constraint in the region growing, it is expected that the proposed ASMF will make a correct estimate for a ramp structure with a gentle slope and for a flat area. However, owing to the limited number of pixels that could be found for the denoising window, the ASMF shows an inferior performance on a steplike edge (i.e., an extremely steep ramp edge). To remedy this problem, the DWMTM filter and the AMF are proposed to be in collaboration with the ASMF. On the basis of the evaluation of Fong et al. ${ }^{14}$ the DWMTM filter has very strong ability in noise reduction and very good edge preservation capability with the implicit assumption of the flat facet model. However, the DWMTM filter is not good in preserving the lines and the corners in an image, inheriting the drawback of the median filter used to determine the reference of the thresholding. In contrast, the AMF has been considered to be very good in line and edge preservation and has strong ability in noise reduction. ${ }^{14}$ It is also expected to be capable of preserving the corners by gathering those pixels with the similar gray levels to the target pixel for the mean computation.

The hybrid filter was devised to apply the ASMF to the flat areas and the ramp structures with gentle slopes, and the DWMTM filter and the AMF to the steplike edges. Because the performance of the AMF is comparable with or better than that of the DWMTM filter in edge, line, and corner preservation, the AMF would be a reasonable choice for the most cases of the steplike edges. When the target pixel is corrupted by the noise to a great extent, the DWMTM filter is adopted in place of the AMF. The reason is,

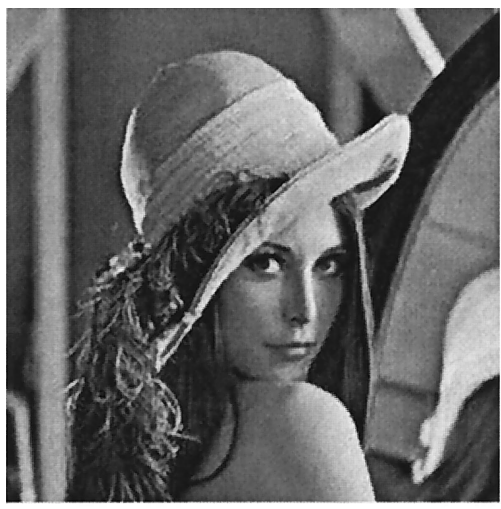

(a)

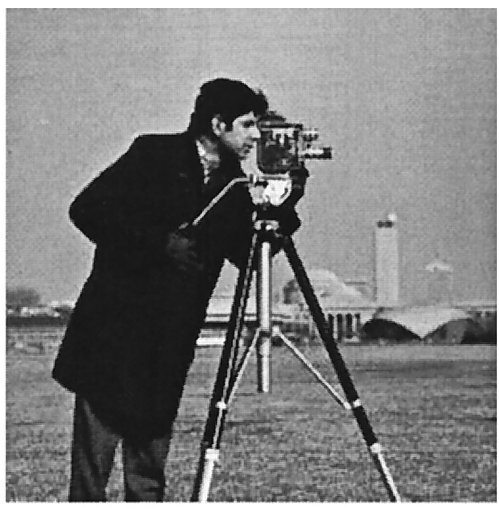

(b)

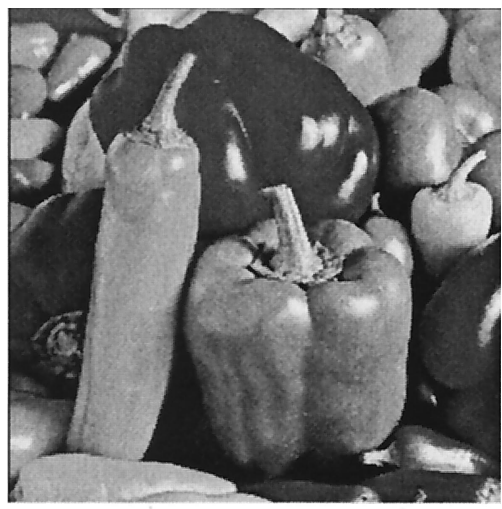

(c)

Fig. 4. Original natural images (a) Lenna, (b) Cameraman, and (c) Peppers. 
Table 1. Parameter Settings Used in the Experiments for the DWMTM Filter, the AMF, the ANNS Filter, the ASMF, and the Hybrid Filter

\begin{tabular}{lll}
\hline \multicolumn{1}{c}{ Filter } & \multicolumn{1}{c}{ Window Size } & \multicolumn{1}{c}{ Threshold } \\
\hline DWMTM & $D L W_{k}=$ best & $T_{\text {dwmtm }}=2 \sigma_{n}$ \\
Filter & $D S W_{k}=\mathrm{DLW}_{k} / 2$ & \\
AMF & $A W_{k}=$ best & $T_{\text {amf }}=3 \sigma_{n}$ \\
ANNS Filter & $Q_{\text {anns }}=$ best & $T_{\text {anns }}=2 \sigma_{d^{\prime}}$ \\
ASMF & $L_{\text {asmf }}=$ best & $T_{\text {asmf }}=1.3 \sigma_{n}$ \\
& $W_{\text {kirsch }}=5$ or 7 & \\
Hybrid Filter & $L_{\text {asmf }}=$ best & $T_{\text {dwmtm }}=2 \sigma_{n}$ \\
& $W_{\text {kirsch }}=5$ or 7 & $T_{\text {amf }}=3 \sigma_{n}$ \\
& $T_{w}=\max \left(L_{\text {asmf }}-4,3\right)$ & $T_{\text {asmf }}=1.3 \sigma_{n}$ \\
& $D L W_{k}=D L W_{k} / 2$ & \\
& $D S W_{k}=D L W_{k} / 2$ & \\
& $A W_{k}=L_{\text {asmf }} / 2$ & \\
&
\end{tabular}

in this case, the AMF would likely result in an erroneous estimate and find a bad reference for the thresholding. In contrast to the AMF, the DWMTM filter uses the median filter to attain a better reference for the thresholding. That is probably why the DWMTM filter is slightly better than the AMF in the capability of noise reduction. ${ }^{14}$

To realize this idea, for each target pixel the hybrid filter first employs the ASMF to find the largest denoising window subject to the symmetry constraint. If the number of pixels in the denoising window exceeds a predefined threshold $T_{w}$, the mean of the gray levels of all pixels in the denoising window is taken as the output of the hybrid filter. Otherwise, the AMF with a $3 \times 3$ pixel window is utilized to determine if

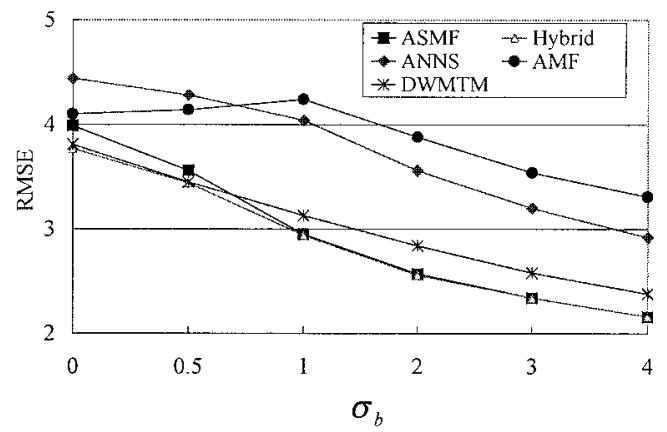

(a)

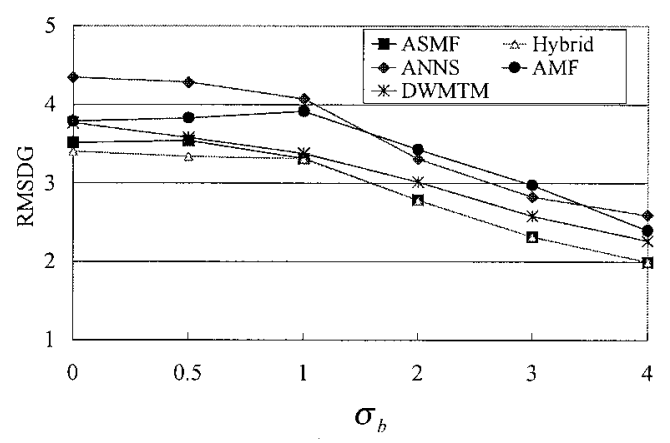

(b)

Fig. 5. (a) RMSEs and (b) the RMSDGs achieved by the five filters with $\sigma_{n}=15$ and $\sigma_{b}=0,0.5,1,2,3$, and 4 .

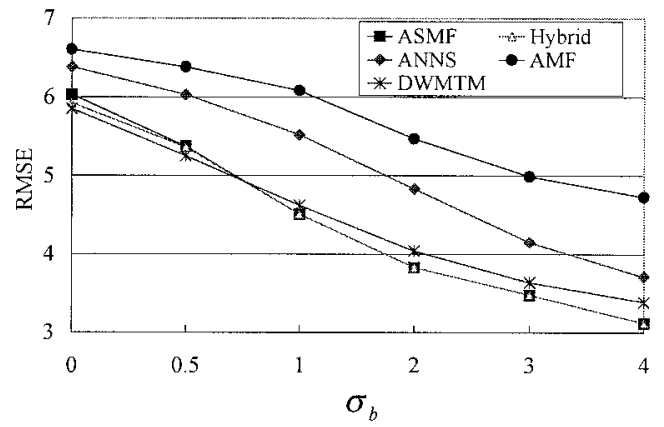

(a)

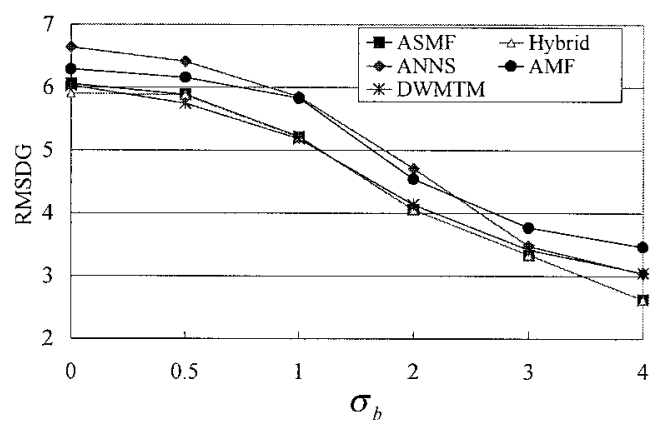

(b)

Fig. 6. (a) RMSEs and (b) the RMSDGs achieved by the five filters with $\sigma_{n}=25$ and $\sigma_{b}=0,0.5,1,2,3$, and 4 .

the target pixel is severely distorted by the noise. If the number of pixels found in the denoising window is fewer than three, the DWMTM filter is used. If not, the AMF is applied.

\section{Experimental Results and Discussions}

Two types of images have been employed to evaluate the ASMF, the hybrid filter, the DWMTM filter, the AMF, and the ANNS filter. One is the synthetic image, and the other is the natural image. The synthetic image is constituted by a set of uniform objects, all of which are blurred by a Gaussian function of a specified standard deviation $\sigma_{b}$. The background of the synthetic image is set to 150 . All objects have different gray levels. By controlling $\sigma_{b}$, the synthetic image serves to simulate the ramp edges of various slopes. For example, Figs. 3(a) and 3(b) show the synthetic images with $\sigma_{b}=0$, i.e., without blurring, and $\sigma_{b}=2$, respectively. However, the natural image provides a true complex image condition, including the real ramp edges, the curved boundaries, the curved surfaces, and the corners, for performance evaluation of all five filters. Figures 4(a)-4(c) give the three natural images used in this study, which are Lena, Cameraman, and Peppers, respectively. The sizes of the synthetic and the natural images are all $256 \times 256$ pixels.

The noise-corrupted versions of the synthetic and the natural image are simulated by superimposing $G\left(0, \sigma_{n}\right)$ onto the image, where $G\left(0, \sigma_{n}\right)$ denotes the zero-mean, additive white Gaussian noises of the specified standard deviation $\sigma_{n}$. The filter perfor- 


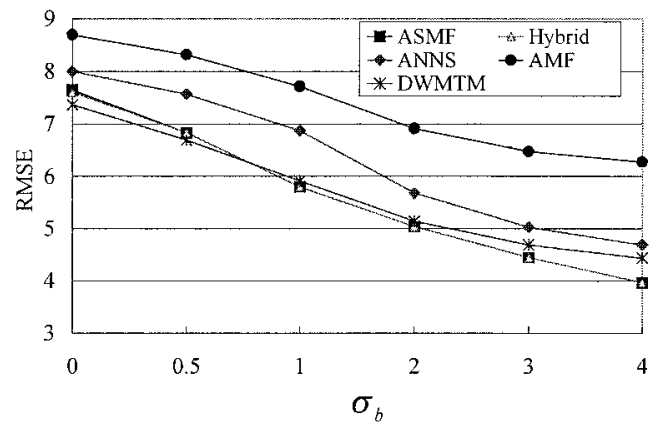

(a)

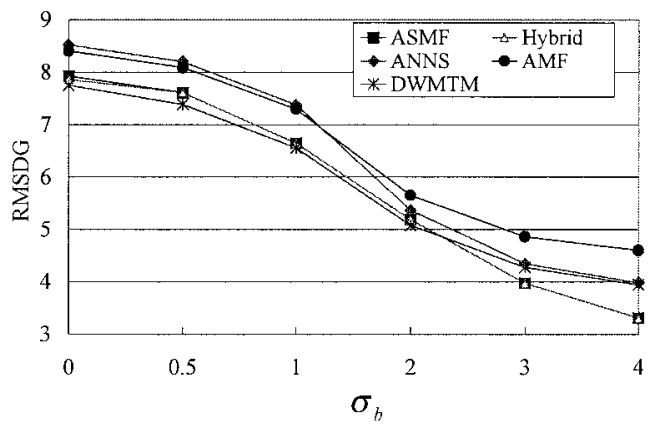

(b)

Fig. 7. (a) RMSEs and (b) the RMSDGs achieved by the five filters with $\sigma_{n}=35$ and $\sigma_{b}=0,0.5,1,2,3$, and 4 .

mance is evaluated on the basis of the root-meansquared error (RMSE) and the root-mean-squared difference of gradient (RMSDG) between the output image (i.e., the denoised image) and the uncorrupted image. Let $f(i, j)$ and $\tilde{f}(i, j)$ denote the gray levels of the uncorrupted image and the output image, respectively, at the position $(i, j)$. Suppose the size of the image is $N_{f} \times N_{f}$. Then the RMSE is defined as

$$
\operatorname{RMSE}=\left\{\frac{1}{N_{f}^{2}} \sum_{i=1}^{N_{f}} \sum_{j=1}^{N_{f}}[f(i, j)-\tilde{f}(i, j)]^{2}\right\}^{1 / 2},
$$

and the RMSDG is defined as

$\mathrm{RMSDG}=\left[\frac{1}{N_{f}^{2}} \sum_{i=1}^{N_{f}} \sum_{j=1}^{N_{f}}\|\nabla f(i, j)-\nabla \tilde{f}(i, j)\|^{2}\right]^{1 / 2}$,

where $\nabla g$ stands for a two-dimensional gradient of $g$ and $\|(u, v)\|=\sqrt{u^{2}+v^{2}}$. Whereas the RMSE measures the overall intensity similarity between the output and the uncorrupted images, the RMSDG emphasizes the variation of the high-frequency image structures of both images. For a more reliable performance evaluation, given $\sigma_{n}$, five corrupted images have been generated for each synthetic or natural image. The efficacy of a filter on removing $G\left(0, \sigma_{n}\right)$ from the a corrupted synthetic or natural image is then defined as the averages of the five RMSEs and the five RMSDGs corresponding to the five corrupted images, respectively.

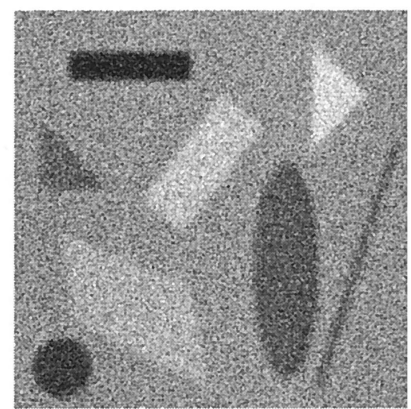

(a)

(c)

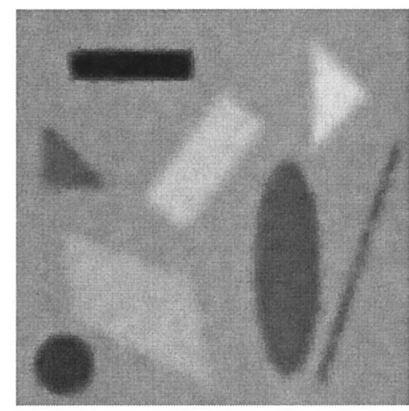

(e)

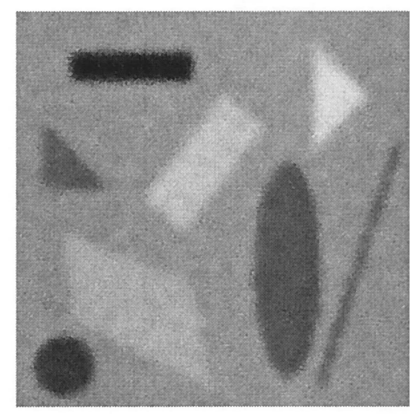

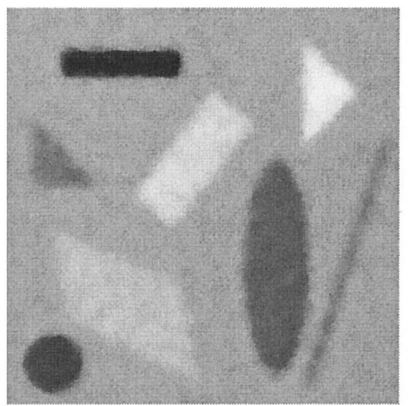

(b)

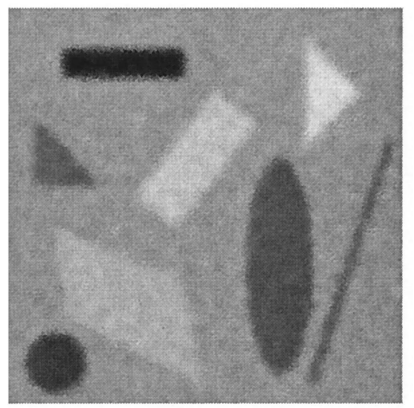

(d)

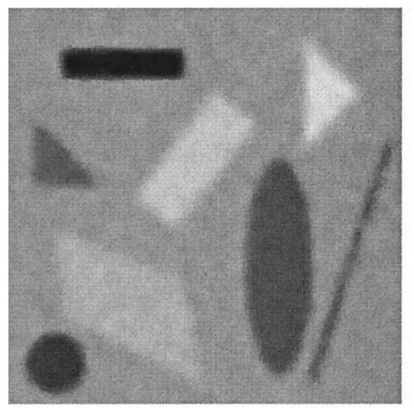

(f)
Fig. 8. (a) Corrupted synthetic image with $\left(\sigma_{b}, \sigma_{n}\right)=(2,25)$, and the denoised image derived with (b) the DWMTM filter, (c) the AMF, (d) the ANNS filter, (e) the ASMF, and (f) the hybrid filter.

To minimize the potential bias that is due to the improper implementation of the filters, the values for the crucial parameters required in the five filters have been assigned on the basis of three principles. One is to use the values suggested by the previous studies. Another is to test a wide range of values and choose the one yielding the best performance in terms of the RMSEs, which tries to ensure that comparisons among these five filters are made on the basis of their best performances. The third is to adopt the empirical values only for the proposed filters, which is to avoid giving any preference to the proposed filters. As a summary, Table 1 lists the parameter settings used in this study. The definition of these parameters may be found in Section 2 . The values for the window size $D S W_{k}$ and the thresholds $T_{\text {dwmtm }}, T_{\text {amf }}$, and $T_{\text {anns }}$ are assigned according 


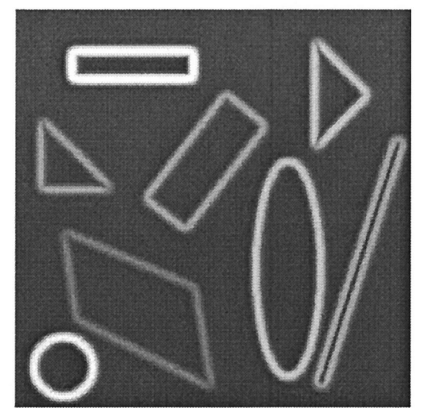

(a)

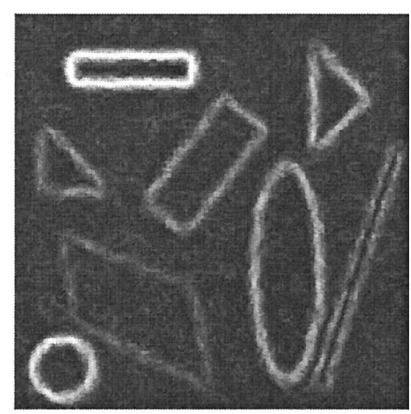

(c)

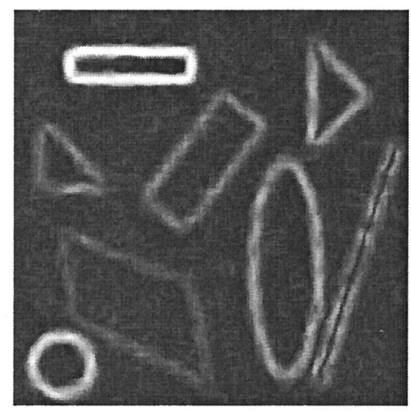

(e)

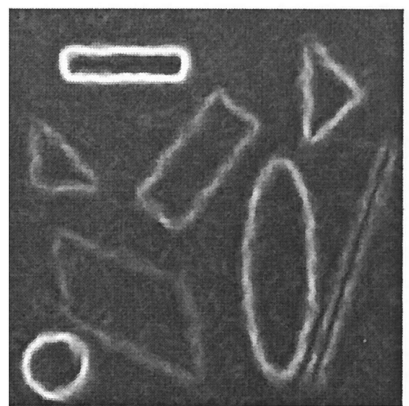

(b)

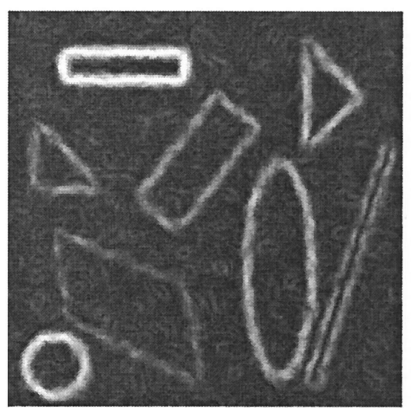

(d)

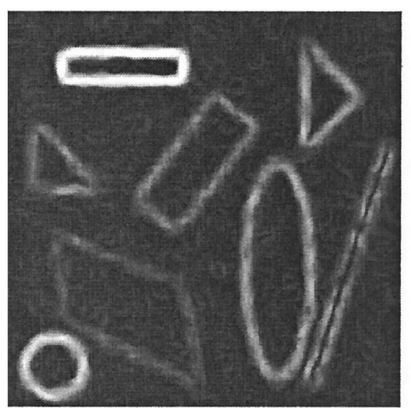

(f)
Fig. 9. (a) Gradient magnitude of the uncorrupted synthetic image with $\sigma_{b}=2$, and the gradient magnitude of the denoised image derived with (b) the DWMTM filter, (c) the AMF, (d) the ANNS filter, (e) the ASMF, and (f) the hybrid filter for the corrupted image $\left(\sigma_{b}, \sigma_{n}\right)=(2,25)$.

to the suggestions made by Ding and Venetsanopoulos, ${ }^{17}$ Fong et al., ${ }^{14}$ and Paranjape et al., ${ }^{11}$ respectively. The values for the window sizes $D L W_{k}, A W_{k}$, $Q_{\text {anns, }}$, and $L_{\text {asmf }}$ are chosen to give the best performances from a wide range of values. The values for the $T_{w}, D L W_{k}$, and $A W_{k}$ used in the hybrid filter and the $T_{\text {asmf }}$ and $W_{\text {kirsch }}$ used in both the ASMF and the hybrid filter are all decided empirically.

\section{A. On the Synthetic Images}

Figures 5-7 give the performance of the five filters on the synthetic images for $\sigma_{n}=15,25$, and 35, respectively. Figures 5(a), 6(a), and 7(a) show the performance in terms of RMSEs, and Figs. 5(b), 6(b), and 7(b) show the performance in terms of RMSDGs.

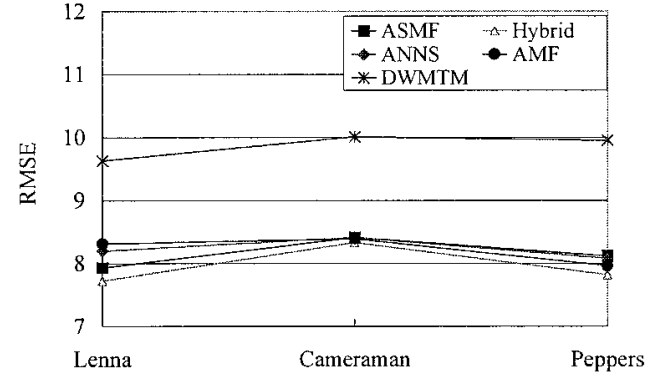

(a)

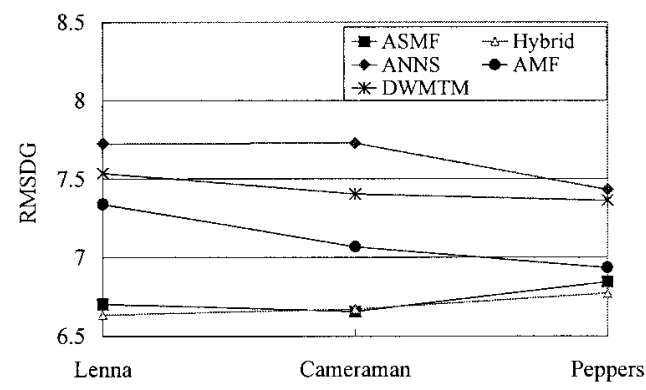

(b)

Fig. 10. (a) RMSEs and (b) the RMSDGs achieved by the five filters with $\sigma_{n}=15$ for the three natural images, i.e., Lenna, Camerman, and Peppers.

For each $\sigma_{n}$, six different $\sigma_{b}$ 's have been considered, i.e., $0,0.5,1,2,3$, and 4 . The small $\sigma_{b}$ 's are to simulate the image structures with steplike edges, and the large $\sigma_{b}$ 's are to account for the slowly varying ramp edges. For visual inspection, for example, Figs. 8 and 9 show the denoised images and the gradient magnitude images derived by the five filters for $\left(\sigma_{b}, \sigma_{n}\right)=(2,25)$, respectively. Figures 8 and 9 demonstrate that the ASMF and the hybrid filter yield more pleasant results on the flat areas and the ramp edges than the other three filters. This observation verifies the theoretical analysis that the ASMF is better than the DWMTM filter, the AMF, and the ANNS filter in terms of estimation errors as given by Eqs. (5)-(10).

The experimental results clearly show that the proposed ASMF and the hybrid filter outperform the DWMTM filter, the AMF, and the ANNS filter on the synthetic images with slowly varying ramp edges. Except the RMSDGs for $\left(\sigma_{b}, \sigma_{n}\right)=(1,35)$ and $(2,35)$, when $\sigma_{b} \geq 1$, the proposed ASMF and the hybrid filter are superior to the other three filters in terms of the RMSE and the RMSDG. It supports the expectation that the gradient similarity criterion and the symmetry constraint for region growing may significantly enhance the estimation accuracy for the image with the ramp edges. However, for the steplike edges, i.e., $\sigma_{b} \leq 0.5$, the ASMF is still better than the AMF and the ANNS filter in most cases but becomes comparable with or slightly worse than the DWMTM filter. The ASMF loses its superiority over the DWMTM filter mainly because the ramp structures 


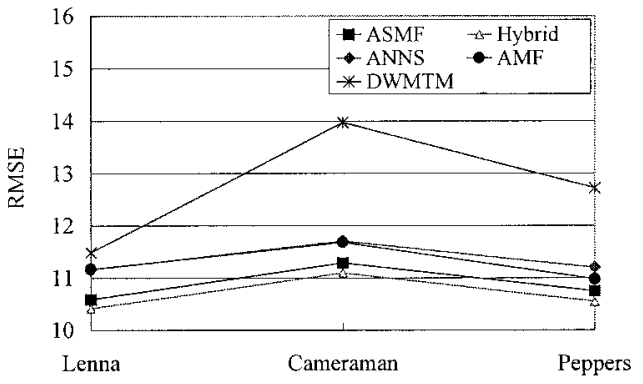

(a)

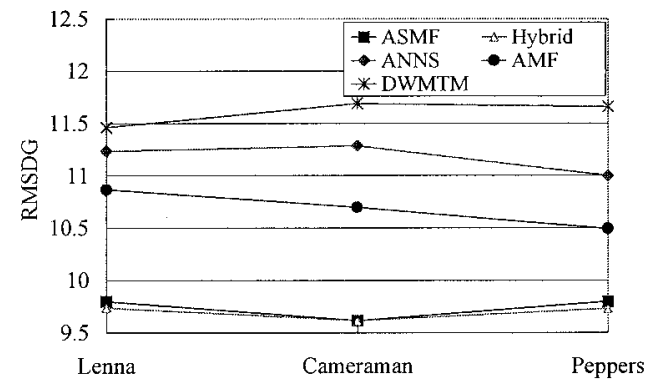

(b)

Fig. 11. (a) RMSEs and (b) the RMSDGs achieved by the five filters with $\sigma_{n}=25$ for the three natural images, i.e., Lenna, Camerman, and Peppers.

become less significant as $\sigma_{b}$ becomes smaller. The reason why the AMF and the ANNS filter are generally inferior to the other three filters for all tested synthetic images may be ascribed to the use of the corrupted pixel intensity as the reference for the similarity measurement, which tends to give a biased average.

By combining the advantages of the proposed ASMF, the DWMTM filter, and the AMF, the performance of the hybrid filter has been shown to be as good as that of the ASMF for $\sigma_{b} \geq 1$ and similar to that of the DWMTM filter with the corners well preserved for $\sigma_{b} \leq 0.5$. The reason is obvious. When $\sigma_{b} \geq 1$, the hybrid filter basically counts on the ASMF for the flat areas and the gentle ramp edges in the synthetic images. When $\sigma_{b} \leq 0.5$, the hybrid filter uses the ASMF on the flat areas and the AMF or the DWMTM filter on the steep ramp edges. Therefore, if the hybrid filter can switch among these three filters effectively, the hybrid filter should be superior to the ASMF, the DWMTM filter, and the AMF filter. However, as the image gets noisier, the judgement on filter switching may become less effective. This accounts for why the hybrid filter has the best performance when $\sigma_{n}=15$ among all cases with $\sigma_{b} \leq 0.5$, as shown in Figs. 5-7. As $\sigma_{n}$ increases, for $\sigma_{b} \leq 0.5$, the performance of the ASMF degrades and becomes worse than that of the DWMTM filter when $\sigma_{n} \geq 25$, as illustrated in Figs. 6 and 7.

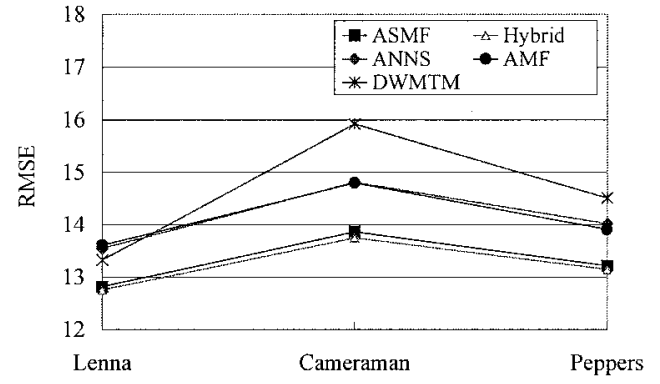

(a)

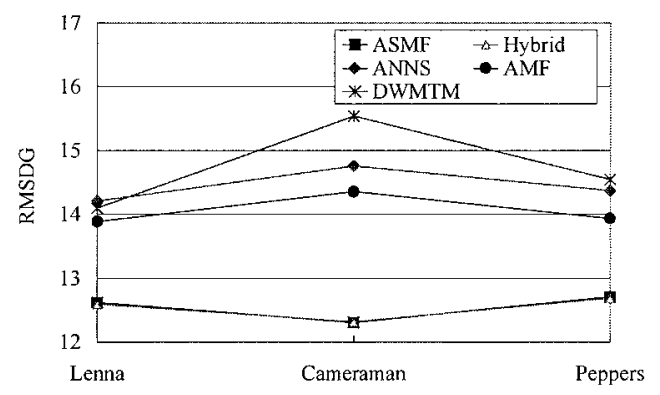

(b)

Fig. 12. (a) RMSEs and (b) the RMSDGs achieved by the five filters with $\sigma_{n}=35$ for the three natural images, i.e., Lenna, Camerman, and Peppers.

\section{B. On the Natural Images}

As on the synthetic images, three different Gaussian noises with $\sigma_{n}=15,25$, and 35 have been added to each natural image. The performances achieved by the five filters are plotted in Figs. $10-12$ for $\sigma_{n}=15$, 25 , and 35, respectively. Figures 10(a), 11(a), and 12(a) show the performance in terms of RMSEs, and Figs. 10(b), 11(b), and 12(b) show the performance in terms of RMSDGs. For visual evaluation, the denoised Lenna, Cameraman, and Peppers images for $\sigma_{n}=25$ are provided in Figs. 13-15, respectively, and their gradient magnitudes are given in Figs. 16-18, respectively. Because the natural images is abundant in the high-order structures such as the ramp structures and the curved surfaces, the experimental results shown in Figs. 10-12 confirm the superiority of the ASMF and the hybrid filter. And the hybrid filter is slightly better than the ASMF. Unlike on the synthetic images, the proposed filters have been shown to be better than the DWMTM filter, the AMF, and the ANNS filter in terms of the RMSEs and the RMSDGs in all cases tested on the natural images. It is probably because the natural images have much richer ramplike structures than the synthetic images.

From Figs. 13-15 one can also find that the ASMF and the hybrid filter have attained much more pleasing results on the originally smooth curved surfaces and flat areas. Some typical examples are the skin surface of the woman's shoulder and face in Lena, the sky and the vertical rod of the tripod in Cameraman, 


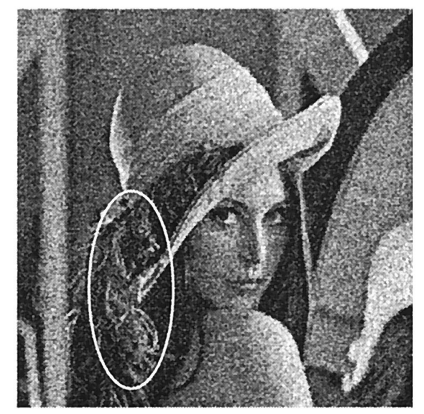

(a)

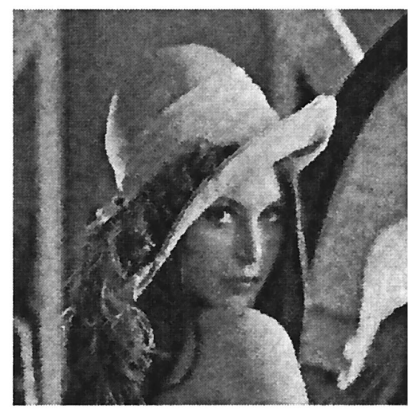

(c)

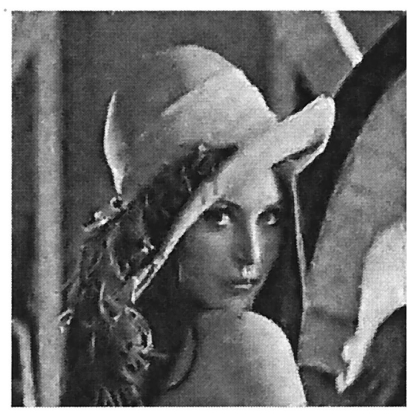

(e)

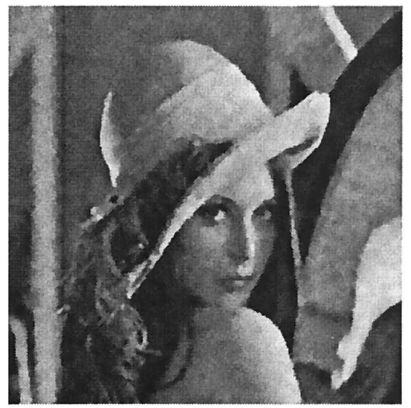

(b)

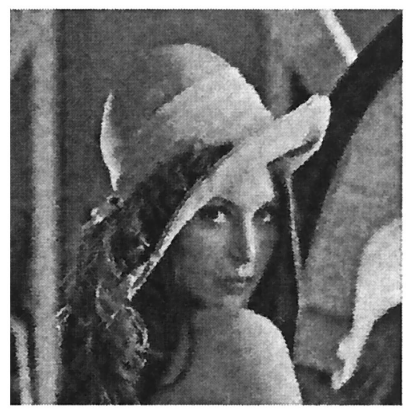

(d)

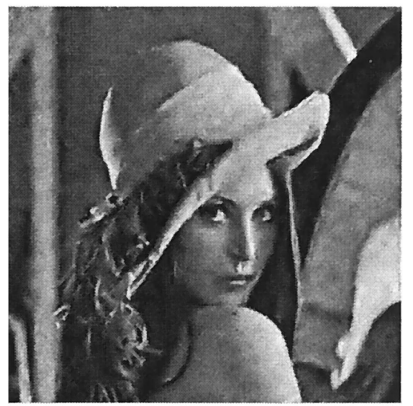

(f)

Fig. 13. (a) Corrupted Lenna image with $\sigma_{n}=25$, and the denoised image derived with (b) the DWMTM filter, (c) the AMF, (d) the ANNS filter, (e) the ASMF, and (f) the hybrid filter. The white ellipse indicates the fine details that may be easily smeared out by the filters.

and the surfaces of various peppers in Peppers. Although the RMSEs achieved by the hybrid filter are a little smaller than those by the ASMF, the differences between the outputs of these two filters are barely perceivable.

Both the ASMF and the hybrid filter were able to preserve the fine details better than the other three filters not only according to the RMSDGs given in Figs. 10(b), 11(b), and 12(b) but also visually appreciable from the denoised images. As examples, one representative fine detail area has been circled out for each natural image with a white ellipse as shown in part (a)'s of Figs. 13-18. It is obvious that the hat decoration strips in Lena, the columns of the building in Cameraman, and the

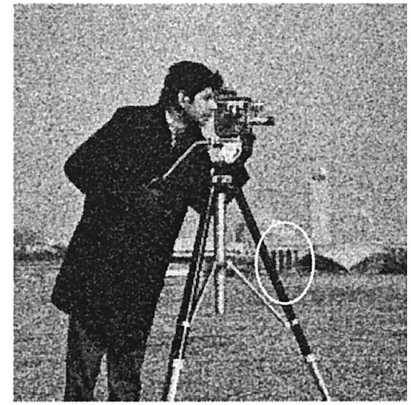

(a)

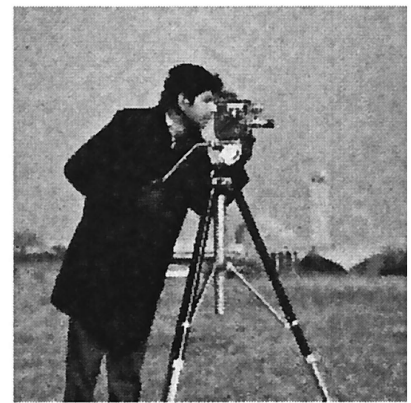

(c)

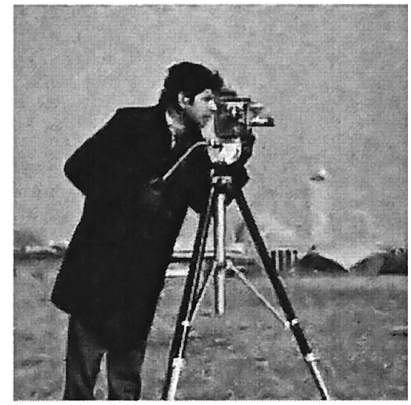

(e)

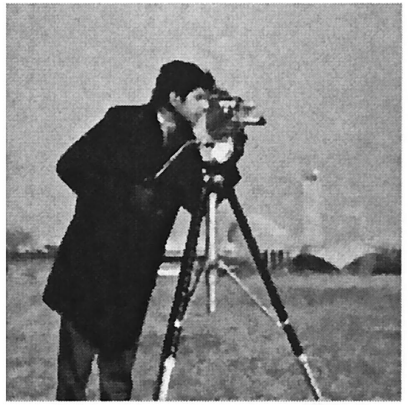

(b)

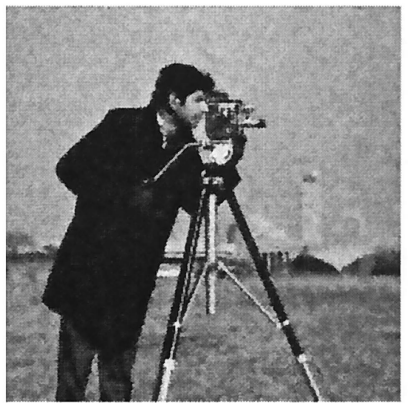

(d)

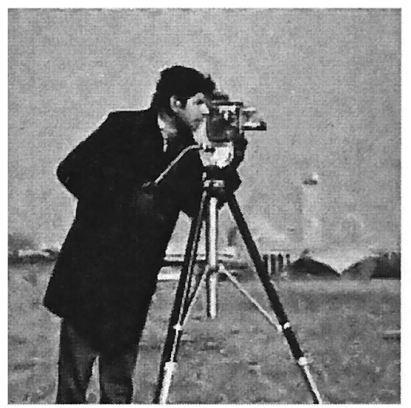

(f)
Fig. 14. (a) Corrupted Cameraman image with $\sigma_{n}=25$, and the denoised image derived with (b) the DWMTM filter, (c) the AMF, (d) the ANNS filter, (e) the ASMF, and (f) the hybrid filter. The white ellipse indicates the fine details that may be easily smeared out by the filters.

bright strips in Peppers have been strikingly well preserved by the ASMF and the hybrid filter. In contrast, these fine details have been severely smeared by the DWMTM filter, the AMF, and the ANNS filter. Especially, the DWMTM filter has destroyed the fine details to a greater extent than the AMF and the ANNS filter owing to the incorporated median filter. For example, only the DWMTM filter failed to preserve the nose tip of the photographer and the bright strip within the leg of the tripod closest to the photographer in Cameraman, as can be seen in Figs. 14(b) and 17(b). That may partially account for why the DWMTM filter ranks the last in terms of the RMSEs on the natural images. 


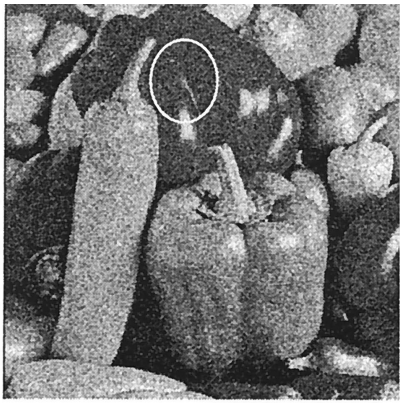

(a)

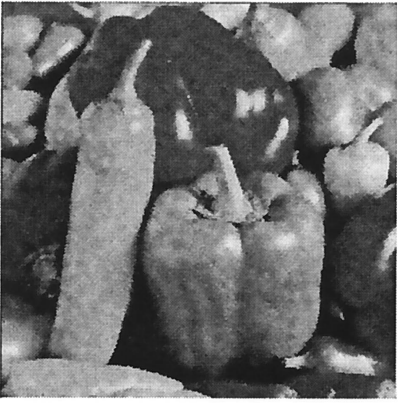

(c)

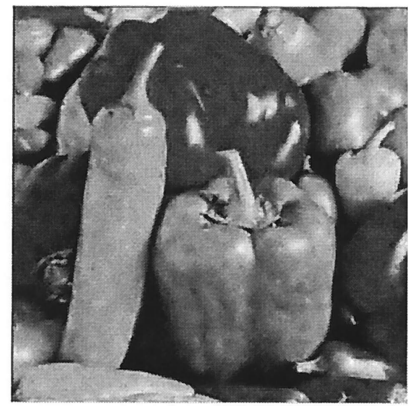

(e)

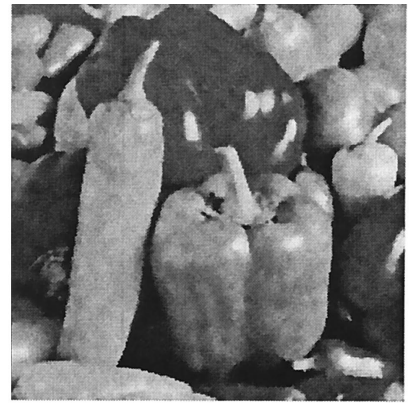

(b)

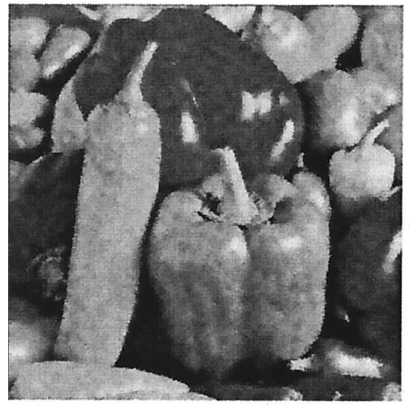

(d)

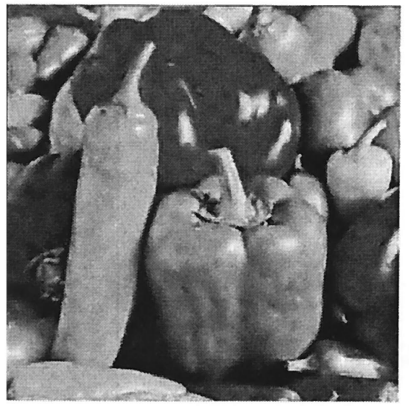

(f)

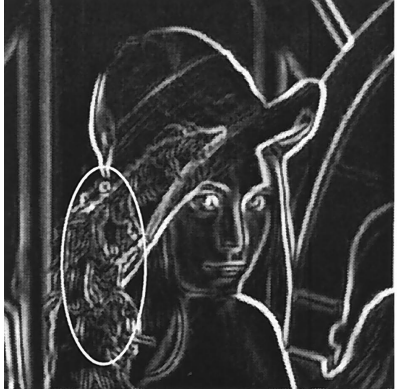

(a)

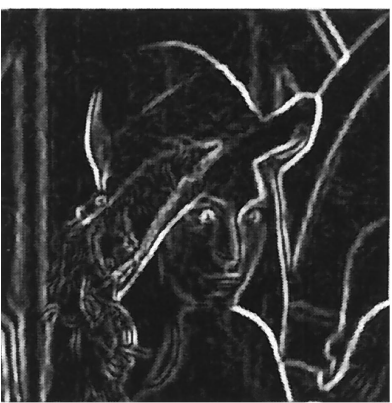

(c)

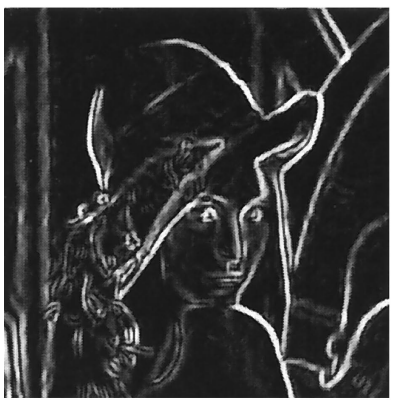

(e)

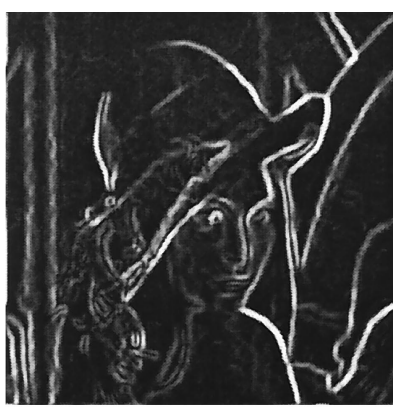

(b)

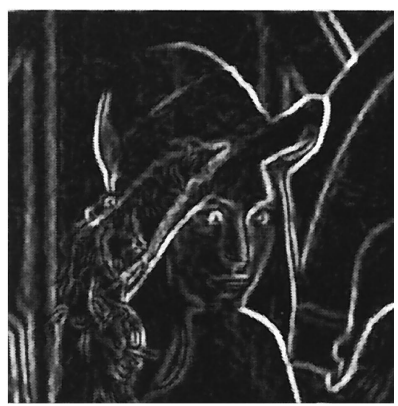

(d)

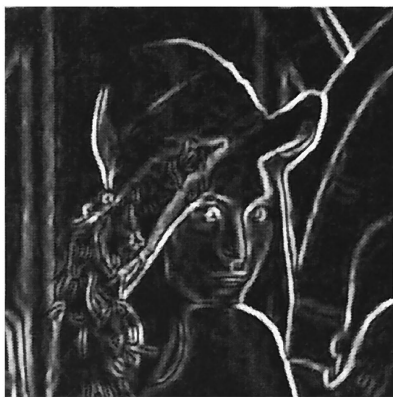

(f)

Fig. 15. (a) Corrupted Peppers image with $\sigma_{n}=25$, and the denoised image derived with (b) the DWMTM filter, (c) the AMF, (d) the ANNS filter, (e) the ASMF, and (f) the hybrid filter. The white ellipse indicates the fine details that may be easily smeared out by the filters.

\section{Conclusions}

Two new noise-reduction algorithms, namely, the ASMF and the hybrid filter, have been proposed in this paper. To ameliorate the deficiency of the conventional noise-reduction algorithms based on the flat facet model in estimating the ramp structures, the ASMF has been designed with the assumption of the slope facet model. A novel region-growing scheme incorporating the gradient similarity criterion and subject to the symmetry constraint has been suggested by the ASMF. The gradient similarity criterion ensures that all pixels in a denoising window are approximately on the same ramp structure or flat area, which has allowed more pixels to be included in a denoising window for a statistically

better estimation. The additional advantage of using the gradient similarity rather than the intensity similarity as the criterion for region growing is that all pixels in a denoising window would be intensity irrelevant. Intensity irrelevancy is an important feature because the mean of all noises in a denoising window will be roughly the same as the mean of each individual noise. With this feature, one may expect that the residual noises remained at all target pixels after the mean of the pixel intensities in the corresponding denoising windows is taken will be approximately the same.

The symmetry property plays a decisive role in ensuring the correctness of the estimation in the expectation sense. It has been made possible by mak- 


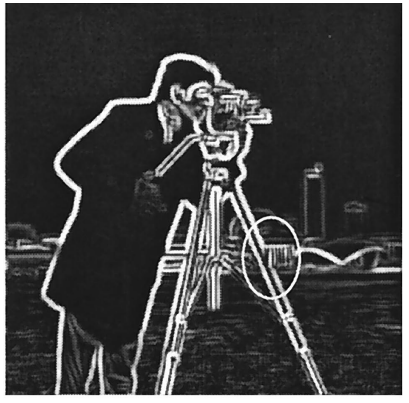

(a)

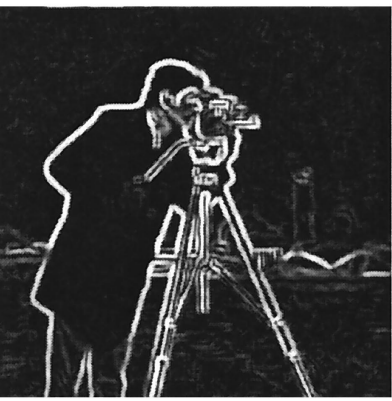

(c)

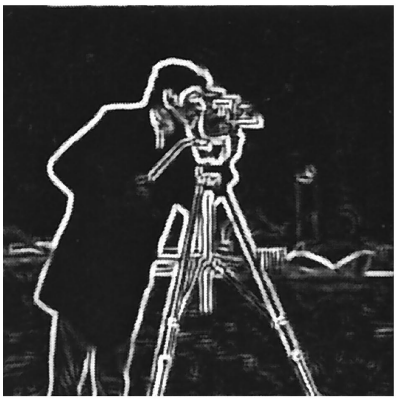

(e)

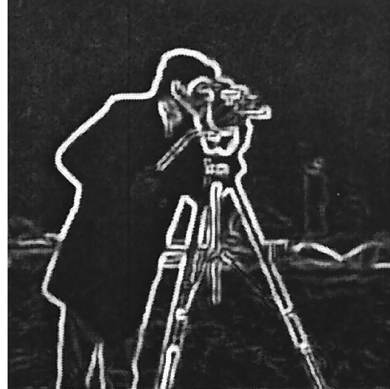

(b)

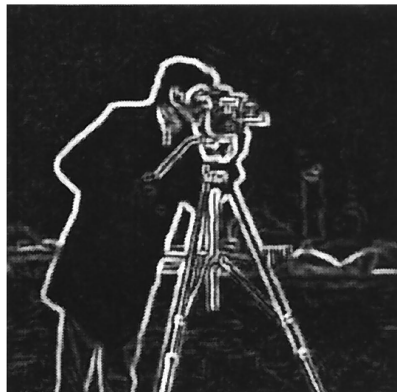

(d)

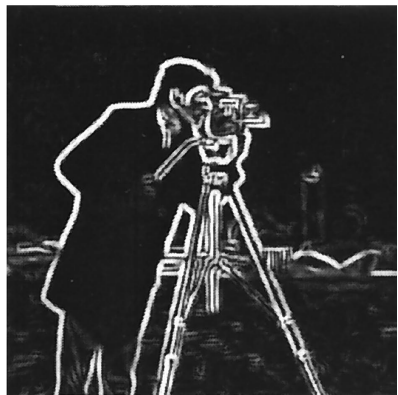

(f)
Fig. 17. (a) Gradient magnitude of the uncorrupted Cameraman image, and the gradient magnitude of the denoised image derived with (b) the DWMTM filter, (c) the AMF, (d) the ANNS filter, (e) the ASMF, and (f) the hybrid filter for the corrupted Cameraman image with $\sigma_{n}=25$. The white ellipse indicates the edges that may be easily smeared out by the filters.

ing sure that all pixels in the denoising window can be paired up such that every pair of pixels are symmetric with respect to the target pixel. As a consequence, the symmetry property guarantees that the mean of the signal portions of all window pixels would be the same as that of the target pixel on a ramp structure or a flat area.

The potential weak point of the ASMF lies in the limited number of pixels that might be found for a denoising window on a step edge, which may lead to a statistically unreliable result. To overcome this problem, the hybrid filter combines the advantages of the ASMF, the DWMTM filter, and the AMF filter in such a way that the latter two are applied to the corners, the lines, and the step edges, whereas the

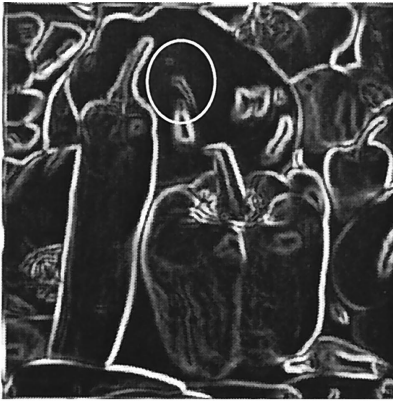

(a)

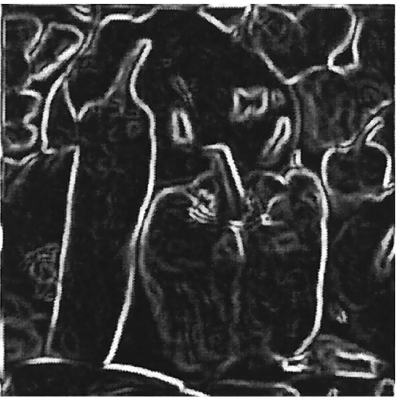

(c)

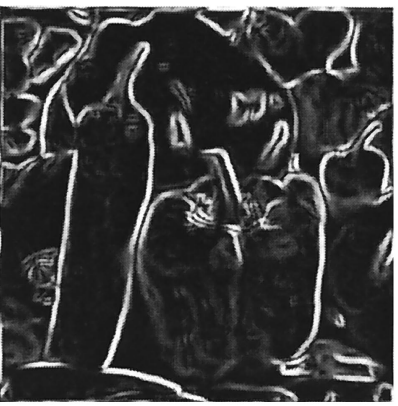

(e)

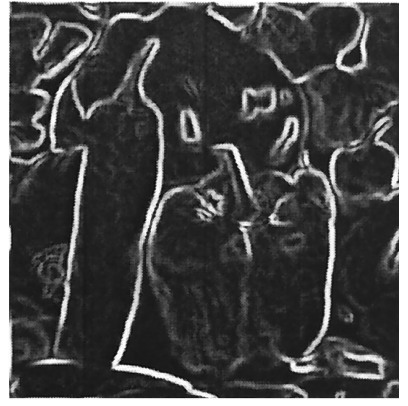

(b)

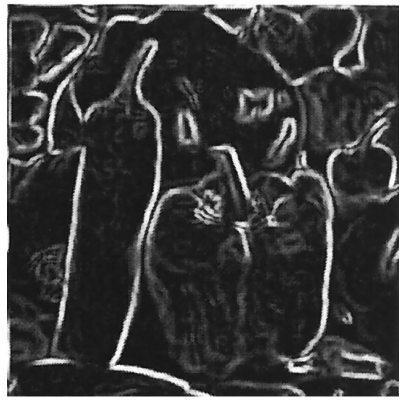

(d)

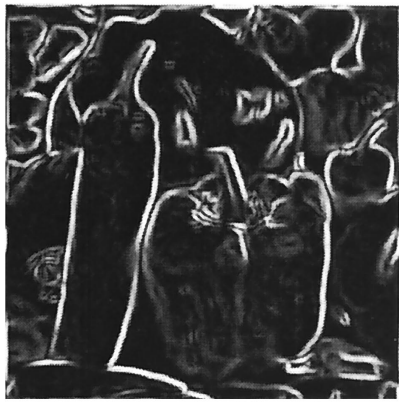

(f)
Fig. 18. (a) The gradient magnitude of the uncorrupted Peppers image, and the gradient magnitude of the denoised image derived with (b) the DWMTM filter, (c) the AMF, (d) the ANNS filter, (e) the ASMF, and (f) the hybrid filter for the corrupted Peppers image with $\sigma_{n}=25$. The white ellipse indicates the edges that may be easily smeared out by the filters.

former one is responsible for the other types of structures.

The proposed two filters, the ASMF and the hybrid filter, have been compared with three conventional filters, the DWMTM filter, the AMF, and the ANNS filter by use of the synthetic and the natural images corrupted by the Gaussian noises of $\sigma_{n}=15,25$, and 35 . On the synthetic images, the proposed two filters outperform the conventional filters in almost all cases when $\sigma_{b} \geq 1$. For $\sigma_{b} \leq 0.5$, the hybrid filter and the ASMF rank the second and the third in most cases, respectively, but only slightly worse than the DWMTM filter. On the natural images, the proposed two filters are superior to the three conven- 
tional filters in all cases tested. Interestingly, the DWMTM filter turns out to be the worst among all filters for the natural images. The proposed filters have not only achieved better performances in terms of the RMSEs and the RMSDGs but also attained visually more pleasing results for almost all of the tested cases.

The authors thank the National Science Council, Taiwan, for its support with the grant number NSC89-2213-E-002-130.

\section{References}

1. R. M. Haralick and L. Watson, "A facet model for image data," Comput. Graph. Image Process. 15, 113-129 (1981).

2. V. Koivunen, "A robust nonlinear filter for image restoration," IEEE Trans. Image Process. 4, 569-578 (1995).

3. I. Pitas and A. N. Venetsanopoulos, "Order statistics in digital image processing,” Proc. IEEE 80, 1893-1921 (1992).

4. C. A. Pomalaza-Raez and C. D. McGillem, "An adaptive, nonlinear edge-preserving filter,” IEEE Trans. Acoust. Speech Signal Process. ASSP-32, 571-576 (1984).

5. S. T. Acton and A. C. Bovik, "Nonlinear image estimation using piecewise and local image models," IEEE Trans. Image Process. 7, 979-991 (1998).

6. D. Geman and C. Yang, "Nonlinear image recovery with halfquadratic regularization," IEEE Trans. Image Process. 4, 932946 (1995).

7. D. C. C. Wang and A. H. Vagucci, "Gradient inverse weighted smoothing scheme and the evaluation of its performance," Comput. Vision Graph. Image Process. 15, 167-181 (1981).

8. J.-S. Lee, "Digital image enhancement and noise filtering by use of local statistics," IEEE Trans. Pattern Anal. Mach. Intell. PAMI-2, 165-168 (1981).

9. Y. H. Lee and S. A. Kassam, "Generalized median filtering and related nonlinear filtering techniques," IEEE Trans. Acoust. Speech Signal Process. ASSP-33, 672-683 (1984).

10. S. R. Peterson and S. A. Kassam, "Edge preserving signal enhancement using generalizations of ordered statistics filtering," in Proceedings of the IEEE International Conference on Acoustics, Speech, and Signal Processing (Institute of Electrical and Electronics Engineers, New York, 1985), pp. 672-675.

11. R. B. Paranjape, T. F. Rabie, and R. M. Rangayyan, "Image restoration by adaptive-neighborhood noise subtraction," Appl. Opt. 33, 2861-2869 (1994).

12. D. Jones and J. Malik, "A computational framework for determining stereo correspondence from a set of linear spatial filters," in Second European Conference on Computer Vision, G. Sandini, ed. (Springer-Verlag, New York, 1992), pp. 395-410.

13. J. Little, "Accurate early detection of discontinuities," in Vision Interface'92 (Canadian Image Processing and Pattern Recognition Society, Toronto, Canada, 1992), pp. 97-102.

14. Y.-S. Fong, C. A. Pomalaza-Raez, and X.-H Wang, "Comparison study of nonlinear filters in image processing applications," Opt. Eng. 28, 749-760 (1989).

15. Y. Boykov, O. Veksler, and R. Zabih, "A variable window approach to early vision," IEEE Trans. Pattern Anal. Mach. Intell. 20, 1283-1294 (1998).

16. R. A. Kirsch, "Computer determination of the constitute structure of biological images," Comput. Biomed. Res. 4, 315-328 (1971).

17. R. Ding and A. N. Venetsanopoulos, "Generalized homomorphic and adaptive order statistic filters for the removal of impulsive and signal-dependent noise," IEEE Trans. Circuits Syst. CAS-34, 948-955 (1987). 